\title{
Management of Nephrotic Syndrome: A Case Report from Lao PDR
}

\author{
Phanekham Souvannamethy \\ Hemodialysis Center and Division of Nephrology, Mittaphab Hospital, Vientiane, Lao PDR
}

\section{Keywords}

Nephrotic syndrome · Kidney biopsy · Iron deficiency anemia. Poor diet

\begin{abstract}
We report the case of a 23-year-old woman with a 2-week history of swelling around the eyes and both legs, and generalized body swelling. She had a history of chronic constipation and poor diet but no fever, recent illnesses, or hematuria. Examination revealed bilateral pedal edema and mild ascites. Laboratory investigations showed low hemoglobin $79 \mathrm{~g} / \mathrm{L}$, low mean corpuscular volume $53 \mathrm{fL}$, thrombocytosis $973 \times$ $10^{9} / \mathrm{L}$, and marked hypochromia and microcytosis, with low iron and ferritin. She had hypoalbuminemia and reduced serum protein (albumin $1.9 \mathrm{~g} / \mathrm{dL}$, globulin $2.8 \mathrm{~g} / \mathrm{dL}$ ) with elevated triglycerides ( $454 \mathrm{mg} / \mathrm{dL}$ ). Although kidney biopsy could not be performed due to a lack of facilities in the country, we made a diagnosis of idiopathic nephrotic syndrome (NS) with iron deficiency anemia secondary to poor diet based on clinical and laboratory findings. The patient was admitted and treated with intravenous methylprednisolone and iron supplements. Antiplatelet therapy was instituted with dipyridamole to prevent thromboembolism from the combination of nephrotic syndrome and thrombocytosis. She was later treated with albumin and furosemide due to elevated blood
\end{abstract}

\section{KARGER}

(C) 2017 S. Karger AG, Basel

E-Mail karger@karger.com

www.karger.com/bpu pressure and worsened edema. The edema resolved and her general condition improved. She was discharged to follow up and has remained stable, requiring no further treatment as of 18 months after admission. Kidney biopsy is important for diagnosis of NS. Diagnosis may be made from clinical and laboratory findings alone in some cases; however, biopsy is required to determine the type of NS and improve further management and treatment outcomes for patients.

(c) 2017 S. Karger AG, Basel

\section{Introduction}

Nephrotic syndrome (NS) is a glomerular disorder typically characterized by gross proteinuria, hypoalbuminemia, hyperlipidemia, and peripheral edema [1]. Diagnosis is generally based on clinical features and investigations including blood tests, renal imaging, and biopsy. In Southeast Asian countries, diagnosis and treatment of renal disease is highly impacted by poor socioeconomic development [2]. In Lao People's Democratic Republic (PDR), facilities for kidney biopsy and renal replacement

See www.karger.com/doi/10.1159/000479576 for an infographic on Country Status.
Phanekham Souvannamethy

Hemodialysis Center and Division of Nephrology

Mittaphab Hospital

Vientiane (Lao PDR)

E-Mail pkhamsouvannamethy@gmail.com 


\section{Patient Assessment}

\begin{tabular}{|c|c|}
\hline July 2015 & 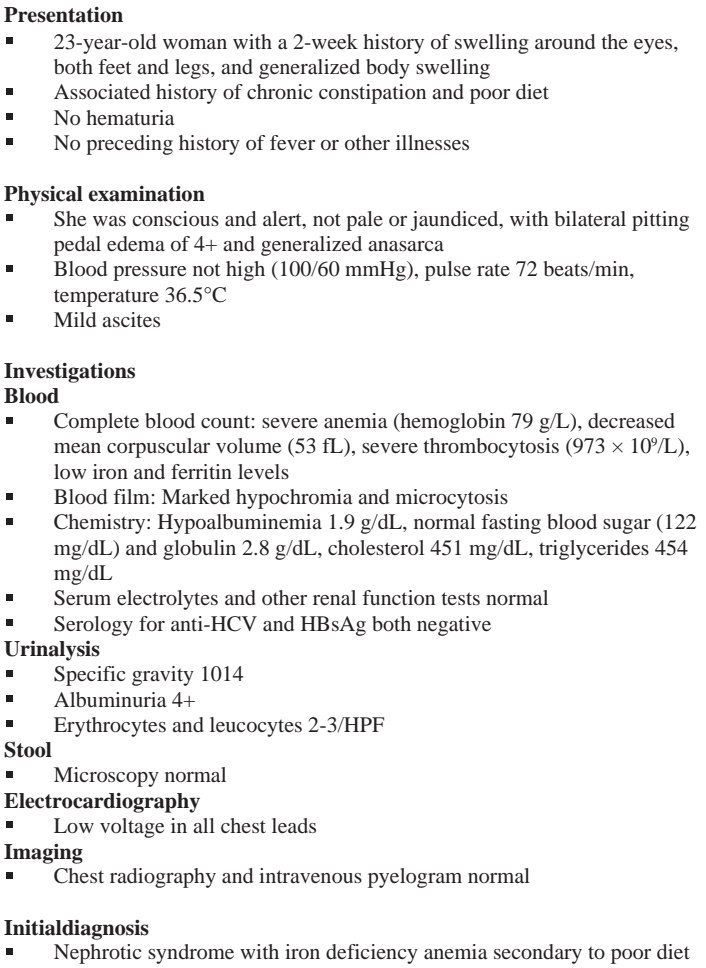 \\
\hline
\end{tabular}

\section{Therapeutic Interventions and Outcome}

\begin{tabular}{|c|c|}
\hline Hospital Day 1 & $\begin{array}{l}\text { Therapeutic interventions } \\
\text { Intravenous methylprednisolone } \\
\text { Iron supplements for the anemia } \\
\text { Dipyridamole anti-platelet agent recommended after nephrology review } \\
\text { to prevent thrombosis }\end{array}$ \\
\hline Hospital Day 2 & $\begin{array}{l}\text { - Albumin and furosemide due to elevated blood pressure and worsened } \\
\text { edema }\end{array}$ \\
\hline Hospital Day 8 & $\begin{array}{l}\text { - } \quad \text { Edema significantly improved } \\
\text { - Switched to oral prednisolone and condition further improved }\end{array}$ \\
\hline
\end{tabular}

\section{Follow-up Assessment of Outcomes}

\begin{tabular}{|c|c|}
\hline $\begin{array}{c}18 \text { months } \\
\text { after admission }\end{array}$ & $\begin{array}{l}\text { Follow-up visits } \\
\text { - Currently returns for routine follow-up visits once a month } \\
\text { Condition remains stable and does not require further treatment }\end{array}$ \\
\hline & $\begin{array}{l}\text { Final diagnosis (Not definitive because kidney biopsy could not be } \\
\text { performed to confirm the type of nephrotic syndrome) } \\
\text { Idiopathic nephrotic syndrome with iron deficiency anemia secondary to } \\
\text { poor diet }\end{array}$ \\
\hline
\end{tabular}

Fig. 1. Timeline showing the clinical course in this patient. 
therapy are both a clinical and economic problem. Although NS can be diagnosed based on clinical findings and results of laboratory investigations alone, biopsy is required to determine the type of NS and thus the most appropriate treatment.

Here, we report a typical case of NS in Lao PDR where the patient presented with clinical features of NS and laboratory findings enabled us to make a diagnosis of NS with iron deficiency anemia. We could not perform kidney biopsy because we do not have the facilities for the procedure in the country yet. Fortunately, the patient made a full recovery following treatment with corticosteroids, iron supplementation, albumin, and diuretics. This report highlights the need for facilities for kidney biopsy and full analysis of biopsy findings in Lao PDR.

\section{Case Report}

A 23-year-old female patient visited our nephrology division in July 2015 with a 2 -week history of swelling around the eyes, both feet and legs, and generalized body swelling. She had no fever, recent illnesses, or hematuria but had a history of chronic constipation and poor diet.

On physical examination, she was conscious and alert, not pale or jaundiced, with bilateral pitting pedal edema of $4+$. There was generalized anasarca. Her blood pressure was not high (100/60 $\mathrm{mm} \mathrm{Hg}$ ), pulse rate was 72 beats/min, and temperature was $36.5^{\circ} \mathrm{C}$. She also had mild ascites. No other abnormalities were detected.

Complete blood count showed low hemoglobin $(79 \mathrm{~g} / \mathrm{L})$ with low mean corpuscular volume (53 fL), elevated platelet count (973 $\times 10^{9} / \mathrm{L}$ ), marked hypochromia and microcytosis on blood film, and low iron and ferritin levels. Urinalysis revealed specific gravity of 1,014 and albumin $4+$, and erythrocytes and leukocytes were both $2-3 /$ HPF. Stool examination was normal. Fasting blood sugar was $122 \mathrm{mg} / \mathrm{dL}$, albumin $1.9 \mathrm{~g} / \mathrm{dL}$, globulin $2.8 \mathrm{~g} / \mathrm{dL}$, cholesterol $451 \mathrm{mg} / \mathrm{dL}$, and triglycerides $454 \mathrm{mg} / \mathrm{dL}$. Serum electrolytes and other renal function tests were all normal. Anti- HCV and HBsAg serology were both negative. There was low voltage in all chest leads on electrocardiography. Chest radiography and intravenous pyelogram were normal. We made a diagnosis of NS with iron deficiency anemia secondary to poor diet. We made a diagnosis of idiopathic nephrotic syndrome (NS) with iron deficiency anemia secondary to poor diet.

Treatment on admission (hospital day 1) consisted of intravenous methylprednisolone because she could not tolerate oral intake. We also administered iron supplements for the anemia. Upon consultation with the nephrology division, the anti-platelet agent dipyridamole was recommended to prevent thrombosis, because the combination of thrombocytosis and nephrotic syndrome increased her risk of a thrombotic event.

On hospital day 2, her blood pressure was elevated and the edema had worsened, so she was treated with albumin and furosemide. By hospital day 8 , the edema had significantly improved. She was switched to oral prednisolone and showed further im-

Management of Nephrotic Syndrome provement. Currently, around 18 months after admission, she returns for routine follow-up visits once a month. So far, her condition has remained normal and she has not required further treatment (Fig. 1).

\section{Discussion}

In this case, we were able to successfully control the peripheral edema with the administration of albumin and diuresis with furosemide. The periorbital edema and leg swelling reduced, and there was a concomitant increase in serum protein levels. This suggests that nephrologists would be capable of managing such patients at some level without access to kidney biopsy in Lao PDR, but also that management will be time-consuming and with no guarantee of success. Our patient also had iron deficiency anemia, which may have been due to poor diet. However, NS is characterized by marked urinary excretion of albumin and other intermediate-size plasma proteins, which leads to impaired metabolism of many plasma proteins and protein-bound substances including erythropoietin and transferrin [2]. This may have been implicated in the iron deficiency anemia in our patient.

Despite a successful outcome in this case, our approach in this case was limited because we could not perform kidney biopsy, as the procedure is currently not performed in our country. This is a major challenge in management of NS and renal diseases in general. For patients who require kidney biopsy, typically we administer captopril for the antiproteinuric effect and refer such patients to Thailand for biopsy, with patients bearing the cost of treatment. We hope to resolve this situation by finding a way for doctors from Lao PDR to train overseas and acquire the skills necessary to perform kidney biopsy. Moreover, we will need to develop facilities for full analysis of biopsy findings. Although kidney biopsy is not always indicated for the diagnosis of NS, particularly when clinical features and laboratory and other investigation findings are suggestive of NS, biopsy helps to determine the type of NS and thus the most appropriate treatment, which enables timely management and improves outcomes for patients.

Another challenge of managing NS and other renal diseases is the nonavailability of medications, and even when available, the drugs are too expensive for patients to afford. Treatment of NS is difficult and time-consuming and younger patients tend to respond better to medication than older patients; this may have contributed to the favorable outcome in the present case. Serum protein lev-

Blood Purif 2017;44(suppl 1):31-34 DOI: 10.1159/000479615 
els do not always increase satisfactorily. Our patient's serum protein levels increased and edema improved following treatment with albumin and furosemide. Despite treatment, many patients do not recover and the disease progresses often resulting in complications such as infections (peritonitis, sepsis), hypovolemic crisis, and thromboembolism (venous or pulmonary embolism) [3]. Thromboembolism is considered to be the most lifethreatening complication of NS after infections [4]. Our patient had severe thrombocytosis, which put her at high risk of developing thromboembolism. However, she was placed on prophylactic dipyridamole to prevent the occurrence of a thrombotic event. She subsequently made a full recovery and requires no further treatment as of 18 months after admission.

In conclusion, we have presented a case of idiopathic NS with iron deficiency anemia successfully managed with corticosteroid, albumin, furosemide, and iron supplementation. We could not perform kidney biopsy but could make a diagnosis based on clinical features and investigations, and fortunately our patient recovered and attends monthly follow-up visits. However, not all pa- tients recover with such treatment. Training and facilities for kidney biopsy and full analysis is needed to enable proper diagnosis and improved management of NS in Lao PDR.

\section{Acknowledgements}

The author thanks Florence Orim, MD, PhD, and Caryn Jones of ThinkSCIENCE, Japan, for medical writing support.

\section{Statement of Ethics}

The patient provided verbal consent, as is common practice in the country, to report her case.

\section{Disclosure Statement}

Writing support, for educational purposes, was funded by $\mathrm{Ni}$ pro Corporation, Japan. Nipro Corporation had no role in the selection of the case, the collection and analysis of the data, or in the preparation of the manuscript.

References

1 KDIGO Clinical Practice Guideline for Glomerulonephritis KDIGO Clinical Practice Guideline for Glomerulonephritis KDIGO Clinical Practice Guideline for Glomerulonephritis. 2012. Available at: http://kdigo.org/ wp-content/uploads/2017/02/KDIGO2012-GN-Guideline-English.pdf. Accessed June 16, 2017.
2 Vaziri ND, Caldwell M, Holbein B, et al: Erythropoietin and transferrin metabolism in nephrotic syndrome. Am J Kidney Dis 2001; 38:1-8.

3 Park SJ, Shin J Il: Complications of nephrotic syndrome. Korean J Pediatr 2011;54:322-328. 4 Kerlin BA, Ayoob R, Smoyer WE. Epidemiology and pathophysiology of nephrotic syndrome-associated thromboembolic disease. Clin J Am Soc Nephrol 2012;7:513-520. 